\title{
Wahrnehmung und Kausalität in den Schriften der Sinnesphysiologen Hermann von Helmholtz, Johannes von Kries und Viktor von Weizsäcker
}

Ein Beitrag zum Verhältnis von Erkenntnistheorie und Wissenschaftstheorie in der Medizin

von Josef Neumann

\section{Einleitung}

Seit der Mitte des 19. Jahrhunderts spricht man von der «naturwissenschaftlichen Medizin». Wilhelm Windelband glaubte, den Beginn dieser jüngsten Epoche der Medizingeschichte auf das Jahr der 31.Jahrestagung der Deutschen Gesellschaft der Naturforscher und Ärzte in Göttingen 1854 datieren zu können ${ }^{1}$. Ob die damals befürwortete und heute noch gültige Denkweise der modernen Medizin als «materialistisch» gekennzeichnet werden darf oder differenziertere Interpretationen verlangt, eines darf als historische Tatsache behauptet werden, daß das Gespräch zwischen Medizin und Philosophie auch nach deren Zuwendung zu den methodischen Prinzipien der Naturwissenschaften nie unterbrochen, vielmehr mit wechselndem Erkenntnisinteresse und unterschiedlicher Intensität fortgesetzt worden ist. Diese Diskussion zwischen Medizin und Philosophie ist bestimmt durch eine grundsätzlich positive Einschätzung der kritischen Philosophie Kants (während der deutsche Idealismus ebenso allgemein als unerlaubte Verselbständigung der spekulativen Vernunft und als Trennung des Denkens von der empirischen Wirklichkeit gesehen wird) und durch eine wechselnde Akzentsetzung zwischen Erkenntnistheorie und wissenschaftstheoretischer Methodenkritik. So kann beispielsweise eine Tradition eingehender philosophischer Arbeit innerhalb der Sinnesphysiologie aufgezeigt werden, die von Hermann von Helmholtz ausging, von dessen Schüler Johannes von Kries um die Jahrhundertwende fortgesetzt wurde und bei Viktor von Weizsäcker, ergänzt durch die Auseinandersetzung mit der Psychoanalyse Sigmund Freuds, zur Begründung der «anthropologischen Medizin» führte, die als Teil der psychosomatischen Medizin bis heute in Deutschland weiterwirkt. 
Hermann von Helmholtz, dessen Denken weitgehend als ablehnende Antwort auf die «Romantische Medizin» zu verstehen ist, der er vorwirft, daß sie einem «falschen Ideal von Wissenschaftlichkeit nachjagte in einseitiger und unrichtig begrenzter Hochschätzung der deductiven Methode», ${ }^{2}$ richtete sein Wissenschaftsverständnis an der mathematischen Darstellbarkeit physikalischer Gegebenheiten aus und handelte aus der Einsicht, daß empirische Forschung durch erkenntnistheoretische Reflexion zu ergänzen, medizinisches Handeln durch wissenschaftstheoretische Methodenkritik zu begründen sei. So frage sich der Arzt angesichts des Kranken,

«ob man selbst Alles gethan habe, was man zur Abwehr des Verhängnisses hätte thun können, und ob die Wissenschaft auch wohl alle Kenntnisse und Hülfsmittel vorbereitet habe, die sie hätte vorbereiten sollen, um zu wissen, daß erkenntnistheoretische Fragen über die Methodik der Wissenschaft auch eine bedrängende Schwere und eine furchtbare praktische Tragweite erlangen können.» ${ }^{3}$

v. Helmholtz, der hier auf das Verhältnis von medizinischer Praxis und wissenschaftstheoretischer Methodenkritik, von Handlungs- und Erkenntnisanspruch hinweist, versteht unter Wissenschaftstheorie die Begründung und Rechtfertigung naturwissenschaftlicher Methoden in der Medizin. Im Vordergrund seiner Erkenntnistheorie hingegen stehen Fragen, die im Kontext empirischer, sinnesphysiologischer Untersuchungen entstanden sind und diese ergänzen. Besonders bedeutsam sind in diesem Zusammenhang Kants Raum- und Zeittheorie sowie die für den Neukantiannismus typische Frage, wie im Affiziertwerden der Sinnesorgane durch äußere Reize und im Zusammenwirken mit cerebralen Funktionen bewußtes Erkennen überhaupt möglich ist.

Auch Johannes von Kries, Schüler von Hermann v. Helmholtz und Karl Ludwig, den sein Nachfolger Paul Hofmann als «Philosoph auf dem Freiburger Lehrstuhl für Physiologie» bezeichnet hat, blieb erkenntnistheoretisch orientiert. Seine philosophische Reflexion gewann aber in dem Maße auch wissenschaftstheoretische Bedeutung, in dem das allein naturwissenschaftlich bestimmte Wissenschaftsverständnis der Medizin zum Ende des 19. Jahrhunderts fragwürdig wurde.

«Der Philosophie rechnen wir all die Fragen zu, auf die wir geführt werden, wenn wir erwägen, welchen letzten, nicht weiter auf anderes zurückführbaren Sinn wir den Sätzen irgendeiner Wissenschaft zuschreiben dürfen, oder aus welchen, keiner weiteren Begründung mehr bedürftigen Überzeugungen wir ihre Berechtigung erweisen können.» ${ }^{4}$ 
v. Kries, den sein Schüler Viktor von Weizsäcker für den «bedeutendsten deutschen Physiologen seiner Generation und für den letzten großen Sinnesphysiologen überhaupt» hielt ${ }^{5}$, hat in seiner Zeit, in der «der folgerichtige Fortgang der Naturwissenschaften aus ihrer eigenen Entwicklung heraus auf die Notwendigkeit geführt hat, sich mit philosophischen Fragen zu beschäftigen», ${ }^{6}$ den Sinn philosophischer Reflexion darin gesehen, den Anspruch der Gewißheit wissenschaftlicher Erkenntnis zu begründen und die Inhalte naturwissenschaftlicher Erkenntnis über den Erfahrungsbereich hinaus zu vervollständigen? .

Demgegenüber leitete Viktor von Weizsäcker, den v. Kries gern als Nachfolger in Freiburg gesehen hätte ${ }^{8}$, die Notwendigkeit einer systematischen philosophischen Reflexion in der Medizin aus dem Anspruch ab, die Praxis der Medizin von Grund auf zu reformieren und ihr Handeln neu zu begründen. Um die Jahrhundertwende war die bisher gültige Handlungslegitimation, die Anwendung naturwissenschaftlicher Methoden auf die Behandlung des Kranken, fraglich geworden, und die Erfahrungen des Ersten Weltkriegs hatten auch in der Medizin einen Reflexionsprozeß in Gang gesetzt, der auf die Notwendigkeit praktischer Reformen hinwies.

\begin{abstract}
«Der Versuch, die Medizin und den ärztlichen Beruf neu zu sehen und noch auf andere Grundlagen als die der exakten Naturwissenschaften zu stellen, nahm seinen Ursprung nicht nur in philosophischen und religiösen Bedürfnissen, sondern in einem ganz aufrichtigen Unbehagen in der Ausübung unseres Berufes. Ich habe den Zwiespalt zwischen Laboratoriumsdenken und Krankensaalhandeln schon berührt. Das Wichtige daran war nicht, wie wir meinten, die Uneinheitlichkeit und Unvollkommenheit; denn Denken und Handeln können nie übereinstimmen. Sondern entscheidend war, wie wir die Doppelseitigkeit unserer Existenz empfanden, nämlich nicht als Bereicherung, sondern als Zerrissenheit, ja als Lüge ... Das Programm der Pathologischen Physiologie war keineswegs abgespielt und in Mißkredit erklärt. Man spürte nur, daß man nicht mehr freudig daran glaubte. Man war sich noch nicht klar darüber, daß der «ursprüngliche Plan, die Behandlung innerer Krankheiten nach den Grundsätzen der Pathologischen Physiologies ... zu gestalten, undurchführbar sei; man fühlte aber, daß die Krise der Medizin eingesetzt hatte.» ${ }^{9}$
\end{abstract}

Auf dem Hintergrund dieser Erfahrungen ist v. Weizsäckers Hauptwerk «Der Gestaltkreis» (1940) einerseits der Versuch einer auf sinnesphysiologisch, experimenteller Forschung aufgebauten Erkenntnistheorie, andrerseits der wissenschaftstheoretisch begründete Entwurf einer neuen Medizin, die den Menschen primär als personales Wesen begreift und von daher die Geltung des Kausalitätsprinzips im medizinischen Denken in Frage stellt. 
Johannes von Kries kommt in der philosphischen Diskussion der Sinnesphysiologen eine besondere Bedeutung zu; denn er nimmt v. Helmholtz' erkenntnistheoretische Fragestellungen auf, setzt diese in Beziehung zur Lehre des psychophysischen Parallelismus Gustav Fechners und entwirft auf dieser Grundlage eine Erkenntnistheorie der naturwissenschaftlichen Medizin, die später unter praktischem Anspruch in der «anthropologischen Medizin» aufgenommen und kritisiert wird, indem v. Weizsäcker sich teilweise den Ansichten von v. Helmholtz wieder nähert.

Mit diesen vorläufigen Hinweisen ist ein Weg vorgezeichnet, auf dem v. Kries' Philosophie in ihren erkenntnistheoretischen und wissenschaftstheoretischen Grundzügen dargestellt werden soll. In einem ersten Abschnitt über das Verhältnis von Empfindung und Wahrnehmung steht v. Kries' Raum- und Zeittheorie im Vordergrund. Diese erkenntnistheoretische Diskussion, in der immer wieder der Vergleich mit entsprechenden Aussagen bei v. Helmholtz und v. Weizsäcker hilfreich sein wird, führt weiter zur Erörterung des Kausalitätsprinzips, in der schließlich der Gegensatz zwischen $\mathrm{v}$. Kries und $\mathrm{v}$. Weizsäcker besonders deutlich zutagetreten wird. Gleichzeitig kann aber gezeigt werden, wie neben dem erkenntnistheoretischen Interesse immer mehr das einer wissenschaftstheoretischen Methodenkritik unter dem Anspruch, die Praxis der Medizin zu verändern, an Bedeutung gewinnt.

\section{Empfindung und Wahrnehmung}

v. Kries unterscheidet die «Empfindung», das unvermittelte, ins Bewußtsein dringende Affiziertwerden der Sinnesorgane durch äußere Reize und die "Wahrnehmung», in der die bewußten Anschauungen und Vorstellungen der Dinge mit der Erfahrung von Raum und Zeit einhergehen. In der Wahrnehmung tritt «jeder Sinneseindruck ebenso unmittelbar wie mit seinen sonstigen Beschaffenheiten auch mit seiner räumlichen Bestimmung ins Bewußtsein». Dabei setzen Bewußtseinsinhalte eine «psychische Verarbeitung des direkt gegebenen Empfindungsmaterials» voraus ${ }^{13}$.

Die Möglichkeit, einzelne Gegenstände im Raum wahrzunehmen und sich gleichzeitig den Raum unabhängig von Dingen vorzustellen, sieht v. Kries in der anatomischen Beschaffenheit und den physiologischen Funktionsweisen der Sinnesorgane begründet. Diese ermöglichen spezifische Empfindungen als Sinnesleistungen, die in hirnphysiologisch erklärba- 
ren Vorgängen weitergeleitet, cerebral verarbeitet werden und als bewußte Wahrnehmungen psychische Empfindungen und Verhaltensweisen bewirken. Dabei ist die anatomische und physiologische Eigenart des Wahrnehmungsapparats bestimmend für die Spezifität der Empfindungen, daß Gegebenheiten so und nicht anders wahrgenommen werden:

«So erzeugt der Sehnerv Licht- und Farbempfindungen, der Geschmacksnerv die Empfindungen des Süßen, Sauren usw. nicht allein bei Erregung durch Licht bzw. bei chemischen Einwirkungen, sondern auch, wenn sie durch mechanische oder elektrische Angriffe in Erregung versetzt werden. Hier ist also die Bedeutung des subjektiven Faktors für die Funktion der Sinne ganz unzweideutig gegeben.» ${ }^{14}$

Gleichzeitig hat auch die Wahrnehmung von Raum und Zeit ihren Grund darin, «daß unsere sinnlichen Wahrnehmungen durchweg in räumlicher Form gegeben sind, und daß eben dies in einer - sei es nun psychologischen oder physiologischen - Beschaffenheit unser selbst seinen Grund hat und seine Erklärung findet.» ${ }^{15}$

Indem v. Kries die «räumliche Form der sinnlichen Wahrnehmung» als ein «durch unsere eigene Natur festgelegtes Merkmal alles Erfahrungswissens» bezeichnet, er spricht auch von der «räumlichen Natur», die «unserem sinnlichen Wahrnehmen als ein dauerndes, unveränderliches Merkmal zukommt», begreift er den Raum einerseits im Sinne Kants als notwendige, in der Natur des Subjekts begründete Anschauung a priori, andrerseits verändert er Kants Apriori-Begriff grundlegend im Sinne einer «psychologisch-genetischen Deutung». ${ }^{16}$

Für Kant sind Raum und Zeit reine Anschauungsformen, Bestimmungen, von denen das Vorstellungsvermögen nie absehen kann, da «wir niemals denken können, daß kein Raum sei, wohl aber, daß in dem selben keine Gegenstände angetroffen werden». Kant fragt ausschließlich nach den in der Natur der Vernunft begründeten logischen Voraussetzungen der Erfahrung mit dem Ziel, Möglichkeiten und Grenzen der Vernunft in der Reflexion ihrer selbst aufzuzeigen.

v. Kries hingegen ersetzt Kants Frage nach den logischen Voraussetzungen des Erkennens in der Weise des Neukantianismus durch die, wie Erkennen überhaupt möglich und von seinen physiologischen und psychologischen Vorbedingungen her zu «erklären» ist ${ }^{17}$. Den von der Sinnesphysiologie in dieser Frage zu leistenden Beitrag sieht v. Kries grundsätzlich als eine Erweiterung und Präzisierung des Kantschen Apriori-Begriffs. Demnach ist die Raumvorstellung nicht nur Kategorie des begreifenden und urteilenden Verstandes, sondern selbst Gegenstand der Wahrnehmung. Das 
heißt, ein psychophysischer Mechanismus wird als materielle Vorbedingung von Erkenntnisvorgängen mit deren logischen Voraussetzungen auf eine Ebene gestellt. Raum und Zeit bleiben damit zwar subjektive Bestimmungen, aber in der Weise, «daß die Raumvorstellung einen in allem Wechsel des psychologischen Geschehens ... sich unverändert erhaltenden, immer wieder in der gleichen Weise aufzuzeigenden Bewußtseinsinhalt darstellt.» ${ }^{18}$

Es ist bereits deutlich geworden, da $\beta$ v. Kries seiner Raum- und Zeittheorie einen psychophysischen Parallelismus zugrunde legt, ein kausal bestimmtes Wirkverhältnis von Soma und Psyche, in dem jeder physiologische Vorgang psychische Empfindungen und Verhaltensreaktionen hervorruft und jedes psychische Erleben in physiologischen Vorgängen seine materielle Grundlage hat.

v. Kries stützt sich dabei auf das von Gustav Fechner aufgestellte «psychophysische Gesetz», das besagt,

«daß die Stärke der Empfindung immer um den gleich Betrag wächst, wenn der Reiz in einem bestimmten Verhältnis vermehrt wird, oder, mathematisch formuliert, daß die Stärke der Empfindung proportional dem Logarithmus des Reizes wachse. Fechner vermutete, daß hierin ein streng gültiges Gesetz der Wechselwirkung zwischen Physischem und Psychischem zu erblicken sei .... ${ }^{19}$

Wenn v. Kries dem Wert psychophysischer Meßformeln auch zurückhaltend gegenüberstand (auf die Argumente zwischen Fechner und v. Kries kann hier nicht eingegangen werden ${ }^{20}$ ), so ist doch zu sagen, daß der psychophysische Parallelismus, das «überall bemerkbare Hineinragen der Sinnesfunktionen in die höheren psychischen Betätigungen, die untrennbare Verknüpfung, die mannigfache Verflechtung, die zwischen diesen beiden Gebieten besteht», ${ }^{21}$ sowohl in seiner sinnesphysiologischen als auch erkenntnistheoretischen Forschung stets vorausgesetzt ist.

Die hier skizzierten Zusammenhänge von Empfindung und Wahrnehmung, Raumvorstellung und psychophysischen Vorbedingungen sollen weiter vertieft werden in einem Vergleich mit den entsprechenden Anschauungen bei v. Helmholtz und v. Weizsäcker.

Der entscheidende Unterschied zwischen v. Helmholtz und v. Kries besteht nicht darin, daß v. Helmholtz seine Erkenntnistheorie weniger streng auf dem psychophysischen Parallelismus aufgebaut hat als v. Kries; er ist vielmehr darin zu sehen, daß sich bereits in v. Helmholtz' Erkenntnistheorie eine Entwicklung anbahnt, in der Raum und Zeit, die Formen sinnlicher Wahrnehmung, immer mehr vom Subjekt abgelöst und zu Wahrnehmungsgegenständen bzw. Erscheinungsweisen gegebener Gegenstände umgedeu- 
tet werden. v. Helmholtz, der empirische Erkenntnis bestimmt sieht im Verhältnis von Wahrnehmung und Bewegung, betrachtet in seiner «empiristischen Theorie» auch den Raum als Wahrnehmungsgegenstand.

«Dennoch wird uns der Raum auch sinnlich erscheinen, behaftet mit den Qualitäten unserer Empfindungen, als das, durch welches hin wir uns bewegen, durch welches hin wir blicken können.» 22

v. Helmholtz deutet den Raum als ein Etwas, als Gegenstand, dessen Wahrnehmung an die konkreten Gegenstände gebunden ist. Subjektive Anschauungsform ist der Raum insofern, als die räumliche Wahrnehmung ein durch anatomische Beschaffenheit und physiologische Funktionsweise bestimmtes Vermögen des Subjekts ist:

«Und eine gegebene, vor aller Erfahrung mitgebrachte Form der Anschauung würde der Raum sein, insofern seine Wahrnehmung an die Möglichkeit motorischer Willensimpulse geknüpft wäre, für die uns die geistige und körperliche Fähigkeit durch unsere Organisation gegeben sein muß, ehe wir Raumanschauung haben können.» ${ }^{23}$

Damit eröffnen sich grundsätzlich zwei Möglichkeiten, die Raumtheorie von v. Helmholtz weiterzudenken, die sich v. Kries und v. Weizsäcker in unterschiedlicher Weise zueigen gemacht haben.

v. Kries hielt sich, wie bereits gezeigt wurde, innerhalb seiner neukantianischen Konzeption eng an Kants Deutung des Raumes als erfahrungsunabhängiger Form sinnlicher Anschauung des Subjekts. v. Weizsäcker hingegen dachte v. Helmholtz' Anschauung von der in der Wahrnehmung der Dinge gegebenen Gegenständlichkeit des Raumes weiter und gelangte so zu einer Ablehnung des erfahrungsunabhängigen Raumbegriffs Kants:

«In der Sinneslehre stellte sich nämlich heraus, daß der ideale Apriorismus von Raum und Zeit, als Anschauungsform jeder möglichen Erfahrung für die Form der Erfahrung, welche wir Wahrnehmung nennen, nicht gilt..» ${ }^{24}$

v. Weizsäcker begreift sinnliche Wahrnehmung als ein physiologisch begründetes Verhältnis von Wahrnehmen und Bewegen. Er lehnt es aber ab, Wahrnehmung «auf die Physiologie der Funktionen zu projizieren, ... da damit ihr Bewußtheitscharakter geopfert würde», ${ }^{25}$ d.h., v. Weizsäcker kehrt sich ab vom psychophysischen Parallelismus. Von daher verbietet es sich für ihn, physiologische Vorbedingungen und logische Voraussetzungen auf eine Ebene zu stellen und das räumliche Vorstellungsvermögen als Folge der Eigenart von Bau und Funktionsweise der Sinnesorgane zu deuten. 
v. Weizsäcker geht in der Kritik der Raumtheorien davon aus, daß es bisher immer als selbstverständlich galt, «daß ein Raum bzw. eine Raumvorstellung da ist, in welcher die Empfindungen 〈lokalisiert` werden; ganz ebenso wie die äußeren Gegenstände als Materie im Raum gedacht werden», und gibt in Anlehnung an v. Helmholtz zu bedenken, «daß das Räumliche nur sekundär entstehe und also an oder in den Gegenständen angetroffen oder erzeugt werden könne.» ${ }^{26}$ Die Unterscheidung zwischen einem objektiven Raum, in dem die Dinge sind, und der Raumwahrnehmung des Subjekts, die von der Wahrnehmung der Gegenstände nicht getrennt werden kann, hält v. Weizäcker für unzulässig und sieht diese aufgehoben in der je aktuellen Situation des Erkenntnisvollzugs, die er als «Ereignis» bezeichnet.

«Wir können Objektives nur im Subjekt, wir können Subjektives nur am Objekt haben. Man kann deshalb ihre Beziehung überhaupt niemals wie zwei einander gegenübergestellte Dinge oder Welten, deren jede für sich Bestand und Stabilität hätte, darstellen. Sondern immer ist nur eine aktuelle und ganz bestimmte Enthaltenheit der Objekte im Subjekt vorhanden.

Für die raumzeitlichen Ordnungen hat aber diese Feststellung folgende Konsequenzen. Sie ebenfalls können nun nicht mehr als bloße Determinierungen oder Eintragungen in einen vorgegebenen (leeren) Raum bzw. ebensolche Zeit aufgefaßt werden. Sondern jede solche Ordnung entspricht einer aktuellen Situation - einem Ereignis. Die Verbindung desselben mit anderen Ereignissen in einer übergreifenden Raumordnung und Zeitfolge ist zunächst selbst nicht mehr als ein neues solches Ereignis und so fort. Man bemerkt, daß eine solcherart entstehende Kette keineswegs als Ganzes im Raum und in der Zeit eingetragen werden kann, sondern in der Geschehens-Fortbildung sind Raum- und Zeitbestimmung jeweils entstanden und wieder aufgehoben, auskristallisiert und in neue Bildungen aufgelöst oder neu aufgenommen worden. Dinge sind dabei nicht im Raum und in der Zeit, sondern Raum und Zeit sind in der Geschehens-Fortbildung entstanden und werden so in oder an den Dingen angetroffen. Die Welt und ihre Dinge sind nicht in Raum und Zeit, sondern Raum und Zeit sind in der Welt, an den Dingen.» ${ }^{27}$

Daraus ergeben sich zwei Konsequenzen: In v. Weizsäckers Erkenntnistheorie sind Raum und Zeit nicht reine Anschauungsformen des Subjekts, sondern Bestimmungen der Gegenstände und deren Erscheinungsweise. Weiter ist zu sagen, die Vorstellungen von Raumordnungen und Zeitfolgen sind nicht im einzelnen Wahrnehmungsakt gegeben, sondern entstehen in der Verbindung mehrerer Erkenntnisinhalte, in der Erinnerung; denn die Erinnerung ist das Vermögen, einmal in der Zeit gegebene Erkenntnisinhalte unabhängig von der gegenwärtigen Wahrnehmung vorzustellen und zueinander in Beziehung zu setzen. Dies soll an einem Beispiel verdeutlicht werden, mit dem wir uns v. Helmholtz' Anschauungen von Raum und Zeit in Erinnerung rufen. 
Es wurde gesagt, daß der Raum erst dann als Raum erkannt werden kann, wenn ein Gegenstand aus unterschiedlichen Positionen im Zusammenwirken von Wahrnehmen und Bewegen angeschaut wird. Da ein Gegenstand aber immer nur aus einer Position im gegenwärtigen Augenblick wahrgenommen werden kann, müssen, damit Raumwahrnehmung entsteht, alle vorhergehenden Wahrnehmungen und ihre Positionen in ihrer zeitlichen Folge in der Erinnerung vorgestellt und mit der eben gegebenen zu der einen Raumwahrnehmung vereinigt werden. Daraus folgt, die Vorstellung des Raumes ist nur möglich in der Zeit. In v. Kries' Worten heißt das:

«Die Zeit ist ... nicht allein die Form, in der das äußere, durch die Sinne erkennbare Verhalten der Umwelt gegeben ist, sondern sie ist auch die Form alles unseres psychischen Erlebens; sie ist nach dem Kantschen Ausdruck die Form unseres <inneren Sinnes〉.» ${ }^{28}$

Sofern Denken ein in physiologischen Vorgängen begründetes psychisches Erleben ist, ist die Zeit die Kategorie, die den Zusammenhang von materiellen physiologischen und psychischen Vorgängen kennzeichnet. Da der psychophysische Parallelismus dieses Verhältnis als das von Ursache und Wirkung begreift, folgt daraus, daß Kausalgeschehen sich in der Zeit vollziehen und nur als zeitliche Folge von Ursache und Wirkung gedacht werden können.

Die erkenntnistheoretische Erörterung von Wahrnehmung und Raumvorstellung hat zur Frage nach dem Verhältnis von Zeit und Kausalität geführt; das bedeutet, es kann von hier aus weiter gefragt werden nach den Voraussetzungen von Naturerkenntnis und medizinischem Denken, von dem v. Kries fordert, es müsse sich aufbauen auf Begriffen, in die der Zeitbegriff eingeht. Der Begriff der Kausalität erfüllt, wie gezeigt wurde, diese Bedingung. Die nun folgende Auseinandersetzung mit dem kausalen Denken in der Medizin wird wiederum im Vergleich der Sinnesphysiologen zeigen, daß sich an dieser Frage die Geister scheiden; denn das Kausalitätsprinzip bzw. der Stellenwert, der ihm im medizinischen Denken zugestanden wird, entscheidet nicht nur über die logische Folgerichtigkeit unterschiedlicher erkenntnistheoretischer Ansätze, sondern drängt zu wissenschaftstheoretischen Stellungnahmen, die medizinisches Handeln legitimieren oder verbieten und ein implizit vorausgesetztes Menschenbild erkennen lassen. 


\section{Kausalitätsprinzip}

In der Frage nach dem Kausalitätsprinzip geht v. Kries von der Erfahrung aus, daß sich der Mensch einerseits im Anspruch freier Selbstbestimmung vorfindet und als Handelnder von sich den «Eindruck der Freiheit, des Sound Anders-Könnens» hat ${ }^{29}$, andrerseits aber Bestimmungen unterliegt, die ihm nicht verfügbar sind. Einerseits erscheint es unvertretbar, das Handeln des Menschen ausschließlich kausal determiniert zu verstehen, andrerseits würde es als ungerechte Beurteilung gelten, würde das Handeln des Menschen allein vom Anspruch der Freiheit her beurteilt, ohne dabei bedingende Begleitumstände zu beachten.

Die Möglichkeit, den aufgezeigten Widerspruch aufzulösen, sieht v. Kries in der Fähigkeit des Menschen gegeben, die Bedingungen seiner Willensentscheidungen und seines Handelns zu erkennen, wenn er auch die Grenze zwischen kausal erklärbarem Bestimmtsein und freier Selbstbestimmung nicht eindeutig ziehen kann. Diesem Lösungsversuch ist entgegenzuhalten, daß der Widerspruch insofern erhalten bleibt, als v. Kries das Denken in psychophysischen Vorbedingungen begründet sieht, die selbst Erkenntnisgegenstand sind und als solche das Kausalitätsprinzip voraussetzen. Da es aber dem Prinzip des psychophysischen Parallelismus widersprechen würde, das Kausalprinzip als reine Kategorie des Denkens anzuerkennen, stellt sich für v. Kries erneut die Frage nach dem Verhältnis von logischer Voraussetzung und psychophysischer Vorbedingung, die gleichbedeutend ist mit der Frage, ob die im Denken erkannten Gesetzmäßigkeiten den in der Natur wirkenden Gesetzen entsprechen und unter welchen Bedingungen diese erkannt werden können.

v. Kries geht davon aus, daß unser Denken nach Gesetzen geordnet ist und sich in der Zeit vollzieht; weiter meint er, daß die «gesetzmäßige Ordnung ein unerläßliches Merkmal jeder Wirklichkeitsvorstellung» ist, und fährt fort:

«Der ... Fall einer völligen Regellosigkeit ist allerdings nicht undenkbar; wäre er aber
gegeben, so würden wir zu etwas, was man eine Erfahrung oder eine Wirklichkeits-
Vorstellung nennen kann, überhaupt nicht gelangen. Gehen wir hiervon aus, so können
wir mit Recht sagen, daß die Gesetzmäßigkeit sim Begriff der Erfahrung lieges. Auch
dieses Verhältnis können wir eine Apriori-Gültigkeit nennen, sofern es sich um ein
Merkmal handelt, das wir unserer Wirklichkeits-Vorstellung unabhängig von dem
speziellen Inhalt unseres Erfahrungswissens zuschreiben dürfen.» ${ }^{30}$

Dabei wird der entscheidende Grund, daß die im Denken erkannten 
Gesetzmäßigkeiten denen der Wirklichkeit entsprechen, in der Möglichkeit der Vorhersage von Gegebenheiten gesehen; denn «soll das Zukünftige irgendwie aus dem Vergangenen ersichtlich sein, so müssen die in der Wirklichkeit enthaltenen Allgemeinheiten von der Art sein, daß sie das Zukünftige auf Grund des Vergangenen eindeutig bestimmen». ${ }^{31}$

Zur Verdeutlichung des Gesagten bietet sich wiederum ein Vergleich mit v. Helmholtz' Erkenntnistheorie an. v. Helmholtz richtet die Frage nach der Entsprechung der im Denken gegebenen und die Ordnung der Wirklichkeit bestimmenden Gesetzmäßigkeiten in gleicher Weise an Philosophie und Naturwissenschaften.

«Die erstere, welche die geistige Seite betrachtet, sucht aus unserem Wissen und Vorstellen auszuscheiden, was aus den Einwirkungen der Körperwelt herrührt, um rein hinzustellen, was der eigenen Thätigkeit des Geistes angehört. Die Naturwissenschaft im Gegentheil sucht abzuschneiden, was Definition, Bezeichnung, Vorstellungsform, Hypothese ist, um rein übrig zu behalten, was der Welt der Wirklichkeit angehört, deren Gesetze sie sucht.» ${ }^{32}$

Auch v. Helmholtz sieht den Widerspruch von logischer Voraussetzung und psychophysischer Vorbedingung, in deren Funktionen das Kausalgesetz vorausgesetzt ist.

«Unsere Empfindungen sind eben Wirkungen, welche durch äußere Ursachen in unseren Organen hervorgebracht werden, und wie eine solche Wirkung sich äußert, hängt natürlich ganz wesentlich von der Art des Apparates ab, auf den gewirkt wird.» ${ }^{33}$

Die Spezifität der Sinneseindrücke wird zwar durch die der Sinnesorgane und der cerebralen Funktionen bestimmt, es darf aber angenommen werden, daß gleiche Reize immer gleiche Empfindungen (v. Helmholtz nennt sie «Zeichen») auslösen. Dadurch entstehe in der Wahrnehmung nicht nur dieser oder jener Gegenstand, sondern, gleichzeitig «die Abbildung der Gesetzmäßigkeit in den Vorgängen der wirklichen Welt». ${ }^{34}$ v. Helmholtz behauptet damit, daß die in der Natur wirkenden Gesetzmäßigkeiten Gegenstand der Wahrnehmung seien. Das gelte auch für das Kausalitätsprinzip, das in der regelmäßigen Beziehung von Gegebenheiten (z.B. zwischen Reiz und Empfindung) erkannt werde; denn, «so ist es gerade das Gesetzmäßige, was sich am regelmäßigsten gleichartig wiederholt, während das zufällig Wechselnde verwischt wird». ${ }^{35}$

Diese Argumentation, die eine Beziehung zwischen Gesetzmäßigkeit und Gewohnheit herstellt und mit der v. Helmholtz der Philosophie der englischen Empiristen, besonders der von David Hume ${ }^{36}$, nahesteht, behauptet letztlich, daß das Kausalitätsprinzip durch Induktion abgeleitet werden könne. 
Dabei wird anerkannt, daß die Methode der Induktion keine letztgültige Gewißheit der Erkenntnis begründen kann. Trotz dieses Widerspruchs von induktiver Methode und Gewißheitsanspruch im Erkennen betrachtet v. Helmholtz das Kausalitätsprinzip nicht als reine kategoriale Form des Denkens, sondern als Gegenstand der Wahrnehmung und bestimmt sein Erkenntnisinteresse dahingehend, die Naturgesetze möglichst vollständig aufzufinden und in ein System wahrscheinlicher Aussagen einzuordnen, dessen Gültigkeit an neuen Gegebenheiten bzw. in der Vorhersage von Gegebenheiten je neu zu bewähren ist.

\begin{abstract}
«Jeder Inductionsschluss stützt sich auf das Vertrauen, dass ein bisher beobachtetes gesetzliches Verhalten sich auch in allen noch nicht zur Beobachtung gekommenen Fällen bewähren werde. Es ist dies ein Vertrauen auf die Gesetzmässigkeit alles Geschehens. Die Gesetzmässigkeit aber ist die Bedingung der Begreifbarkeit. Vertrauen in die Gesetzmässigkeit ist also zugleich Vertrauen auf die Begreifbarkeit der Naturerscheinungen. Setzen wir aber voraus, dass das Begreifen zu vollenden sein wird, daß wir ein letztes Unveränderliches als Ursache der beobachteten Veränderungen werden hinstellen können, so nennen wir das regulative Princip unseres Denkens, was uns dazu treibt, das Causalgesetz. Wir können sagen, es spricht das Vertrauen auf die vollkommene Begreifbarkeit der Welt aus.» ${ }^{37}$
\end{abstract}

Daraus folgt, da $\beta$ das Kausalitätsprinzip als das alle Naturvorgänge beherrschende Gesetz angesehen wird, dessen Geltung sich auch auf die funktionalen Mechanismen der psychophysischen Vorbedingungen des Denkens erstreckt. Diesen erkenntnistheoretischen Determinismus, in dem kein Raum mehr bleibt für den Begriff «Freiheit», führt v. Helmholtz konsequent weiter, indem er auch das menschliche Handeln ausschließlich kausal begründet. Damit findet seine Erkenntnistheorie ihre folgerichtige Weiterführung in einem wissenschaftstheoretischen Konzept, in dem das Verhältnis von Erkennen und Handeln mit dem Begriff «Anwendung» gekennzeichnet und als Kausalverhältnis bestimmt ist.

Unter der Voraussetzung des Kausalitätsprinzips und der Wahrnehmbarkeit der Gesetzmäßigkeiten in der Natur werden auf dem Wege der Induktion Aussagen über die Beziehungen zwischen Gegebenheiten unter dem Anspruch von Notwendigkeit und Allgemeingültigkeit abgeleitet («Korrelation»); d.h. es wird beispielsweise in wenn-dann-Aussagen festgelegt, unter welchen Bedingungen sich Gegebenheiten im Menschen so verändern, daß sein Zustand als «krank» bezeichnet werden muß. Gleichzeitig ist auch der umgekehrte logische Weg möglich. Es kann aufgrund der stets vorausgesetzten Wirklichlichkeit kausaler Gesetzmäßigkeiten vorher- 
gesagt werden, unter welchen Bedingungen bestimmte Gegebenheiten eintreten werden. Das bedeutet, die Absolutsetzung des Kausalgesetzes ermëglicht die Verfügbarkeit von Gegebenheiten - v. Helmholtz formuliert das so:

«Das Begreifen ... ist die Methode, mittelst deren unser Denken die Welt sich unterwirft, die Thatsachen ordnet, die Zukunft voraus bestimmt. Es ist sein Recht und seine Pflicht, die Anwendung dieser Methode auf alles Vorkommende auszudehnen, und wirklich hat es auf diesem Wege schon grosse Ergebnisse geerntet. Für die Anwendbarkeit des Causalgesetzes haben wir aber keine weitere Bürgschaft, als seinen Erfolg.» ${ }^{38}$

Damit weist v. Helmholtz auch auf die Möglichkeit hin, aufgrund der Vorhersagbarkeit von Gegebenheiten kausal begründete Handlungsanweisungen abzuleiten, die in wenn-dann-Beziehungen sagen, in welcher Weise Gegebenheiten verändert werden müssen, damit bestimmte intendierte Gegebenheiten eintreten. Damit ergibt sich aus der Verfügbarkeit und Vorhersagbarkeit von Gegebenheiten die Möglichkeit, die Beziehung von Erkennen und Handeln als ein lineares Anwendungsverhältnis zu beschreiben. Dieses ist dadurch charakterisiert, daß der Schritt vom Erkennen zum Handeln, d.h. die Handlungslegitimation, ausschließlich kausal als «Machbarkeit» begründet wird. Handeln, das sich allein auf kausale Verfügbarkeit von Gegebenheiten und Machbarkeit gründet, ist damit bestimmt als «Technik».

Aus v. Helmholtz' deterministischer Begründung medizinischen Denkens und Handelns haben sich seine Nachfolger in der Sinnesphysiologie nur schwer befreien können. v. Kries, der die Zeit gekommen sah, die Methoden und Inhalte der Naturwissenschaften durch die philosophische Reflexion zu ergänzen und zu vervollständigen, fand keinen Weg aus dem Determinismus und konnte mit dem Hinweis, der Mensch sei in der Reflexion seiner selbst fähig, sich seiner Bedingtheit bewußt zu werden, dem Anspruch des Menschen auf freie Selbstbestimmung nicht gerecht werden. Indem er den psychophysischen Parallelismus konsequenter als v. Helmholtz in seine Erkenntnistheorie einband, wurde das Verhältnis zwischen den logischen Voraussetzungen des Denkens und den psychophysischen Vorbedingungen zum unlösbaren Problem und eine deterministische Bestimmung des Denkvermögens im Grund unausweichlich. In diesem Sinne bestimmt er das «Merkmal der Erfahrung»:

«Zwischen dem in irgend einem Zeitpunkt bestehenden Wirklichkeits-Verhalten und den im gleichen Augenblick sich als notwendige Folge desselben entwickelnden Veränderun- 
gen besteht ein allgemeiner, die Veränderungen als Funktion des Verhaltens (im mathematischen Sinne) eindeutig bestimmender Zusammenhang. Es wird gestattet sein, für das in diesem Satze Ausgedrückte den Namen des Kausal-Prinzips in Anspruch zu nehmen. Es besagt, wie man sieht, einen allgemein angebbaren Zusammenhang zwischen Verhalten und Veränderung, läßt aber die besondere Art dieses Zusammenhanges offen.» ${ }^{39}$

Erst Viktor von Weizsäcker, auf dessen Lehre hier nur hingewiesen werden kann $^{40}$, widersprach v. Helmholtz' Determinismus entschieden und setzte ihm das Konzept des Gestaltkreises entgegen, in dem er aufzuzeigen versuchte, daß das Kausalgesetz im Bereich des Lebendingen kein angemessenes Erkenntnis- und Handlungsprinzip sei. Dabei schien ihm die Überwindung des kausalen Denkens in der Medizin nicht primär aus erkenntnistheoretischen Überlegungen geboten, sondern aus praktischen Erfahrungen im Arzt-Patient-Verhältnis, die ihn dazu führten, die anthropologische Grundprämisse der freien Selbstbestimmung des Menschen anzuerkennen und zum Paradigma medizinischer Wissenschaft und Praxis zu erheben.

Der Vergleich erkenntnistheoretischer und wissenschaftstheoretischer Aussagen in den Schriften der Sinnesphysiologen v. Helmholtz, v. Kries und v. Weizsäcker haben damit zu Ergebnissen geführt, die in folgender Weise zusammengefaßt werden können:

Helmholtz sah sich noch in der Situation, die naturwissenschaftliche Methode nach außen hin verteidigen zu müssen. Die Notwendigkeit, ihre Bedeutung auch für das medizinische Handeln zu erweisen, führte ihn, ausgehend von seinen erkenntnistheoretischen Studien, dazu, das Verhältnis von Erkennen und Handeln zu reflektieren, das er aber auf der Grundlage seiner «empiristischen Theorie» ausschließlich deterministisch bestimmte.

Sein Schüler v.Kries erkannte zwar, daß der Determinismus dem Anspruch freier Selbstbestimmung des Menschen nicht gerecht wird, fand aber letztlich keinen Weg, mit Hilfe der neukantianischen, vom psychophysischen Parallelismus bestimmten Philosophie das kausale, deterministisch ausgerichtete Denken der Medizin im Sinne der anthropologischen Grundprämisse freier Selbstbestimmung zu überwinden.

Erst v. Weizsäcker setzte sich in konsequenter Weise mit diesem Problem auseinander und versuchte, das kausale Denken der Medizin durch das Konzept vom Gestaltkreis zu überwinden. Dabei wurde sein Denken durch praktische Erfahrungen angeregt und hatte die Veränderung medizinischer Praxis im Sinne einer Einführung des Subjekts in die Medizin zum Ziel. 
Damit kann zusammenfassend gesagt werden, daß die wissenschaftstheoretische Reflexion in besonderer Weise durch zwei Faktoren angestoßen wird. Die Notwendigkeit, medizinisches Handeln zu legitimieren, setzt die Reflexion der als wissenschaftlich anerkannten Methoden voraus und führt weiter zur Frage nach dem Menschenbild, das in einer bestimmten Handlungslegitimation implizit vorausgesetzt wird. Daraus wird einsichtig, daß die wissenschaftstheoretische Diskussion der Sinnesphysiologen ihren Grund hatte im Verhältnis von Kausalität und Freiheit.

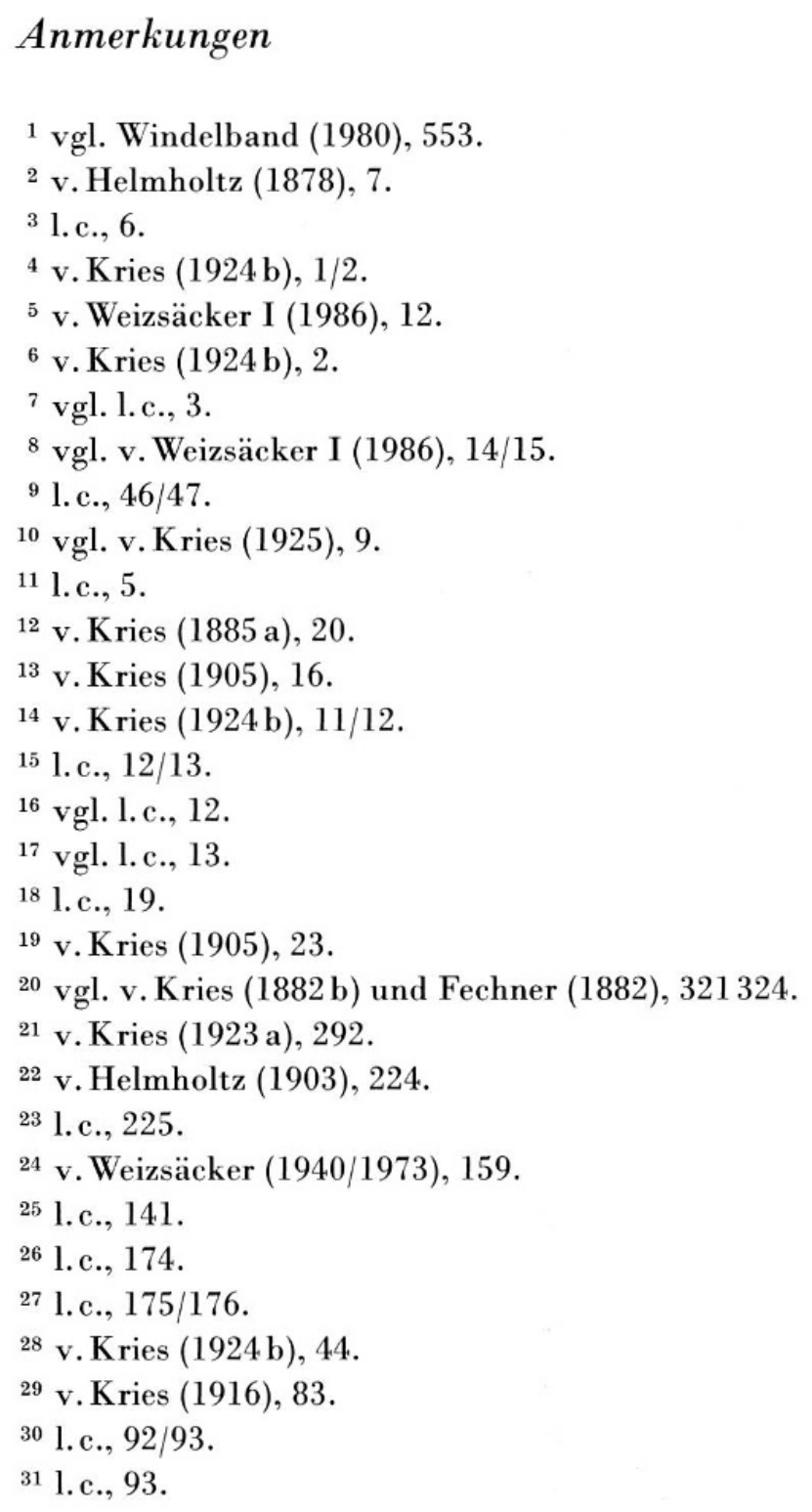


${ }^{32}$ v. Helmholtz (1903), 218.

${ }^{33}$ l.c., 222 .

${ }^{34}$ vgl. l.c., $222 / 223$.

${ }^{35}$ l.c., 232.

${ }^{36} \mathrm{vgl}$. Hume (1984), 3842.

37 v. Helmholtz (1903), 243.

${ }^{38}$ l.c., 243.

${ }^{39}$ v. Kries (1916), 93/94.

\section{Literaturverzeichnis}

1. Bärthlein, Karl (Hrsg.): Zur Geschichte der Philosophie. 2.Band: Von Kant bis zur Gegenwart. Würzburg 1983.

2. Du Bois-Reymond, Emil: Culturgeschichte und Naturwissenschaft. Vortrag gehalten am 24. März 1877 im Verein für Wissenschaftliche Vorlesungen zu Köln. Leipzig 1878.

3. Fechner, Gustav Theodor: Elemente der Psychophysik. 2 Bde. Leipzig 1860.

4. Fechner, Gustav Theodor: Revision der Hauptpuncte der Psychophysik. Leipzig 1882.

5. Helmholtz, Hermann von: Das Denken in der Medicin. Rede gehalten zur Feier des Stiftungstages der militärischen Bildungs-Anstalten am 2. August 1877. Berlin 1878.

6. Helmholtz, Hermann von: Die Thatsachen in der Wahrnehmung. Rede gehalten zur Stiftungsfeier der Friedrich-Wilhelms-Universität zu Berlin 1878. In: Vorträge und Reden. 2. Bd. Braunschweig 1903.

7. Hoffmann, Paul: Johannes von Kries. Ein Philosoph auf dem Freiburger Lehrstuhl für Physiologie. In: Berliner Medizin 9 (1957) 187-192.

8. Hume, David: Eine Untersuchung über den menschlichen Verstand. Übers. von Raoul Richter. Mit einer Einleitung hrsg. von Jens Kulenkampff. Hamburg 1984.

9. Kant, Immanuel: Kritik der reinen Vernunft. 1. und 2. Auflage. Nach der ersten und zweiten Original-Ausgabe neu herausgegeben von Raymund Schmidt. Hamburg 1956.

10. Kries, Johannes von: Gedächtnisrede auf Otto Funke bei dessen akademischer Todtenfeier am 2. Juli 1881. Freiburg 1881.

11. Kries, Johannes von: Die Gesichtsempfindungen und ihre Analyse. In: Arch.f. Physiol. Suppl. (1882 a) 1-178.

12. Kries, Johannes von: Über die Messung intensiver Größen und über das sogenannte psychophysische Gesetz. In: Vierteljahresschr. f. wiss. Philosophie 6 (1882 b) 257-295.

13. Kries, Johannes von: Hermann von Helmholtz. In: Dt. med. Wochenschr. 35 (1891) 1025-1027.

14. Kries, Johannes von: Über Real- und Beziehungsurteile. In: Vierteljahresschr. f. wiss. Philosophie 16 (1892) 253-288.

15. Kries, Johannes von: Carl Ludwig. Freiburg/Leipzig 1895 a.

16. Kries, Johannes von: Über die Natur gewisser mit psychischen Vorgängen verknüpfter Hirnzustände. In: Zeitschr. f. Psychol. 8 (1895b) 1-33.

17. Kries, Johannes von: Über die materiellen Grundlagen der Bewußtseins-Erscheinungen. Freiburg im Breisgau 1898. 
18. Kries, Johannes von: Zur Psychologie des Urteils. In: Vierteljahressehr. f. wiss. Philosophie 23 (1899) 1-48.

19. Kries, Johannes von: Zur Psychologie der Sinne. In: W. Nagel (Hrsg.): Handbuch der Physiologie des Menschen. 3. Bd. Braunschweig 1905.

20. Kries, Johannes von: Logik. Grundzüge einer kritischen und formalen Urteilslehre. Tübingen 1916.

21. Kries, Johannes von: Über Entstehung und Ordnung der menschlichen Bewegungen. Rede gehalten am 26. Januar 1918 bei der von der Universität Freiburg veranstalteten Kaisers-Geburtstags-Feier. Freiburg im Breisgau/Leipzig 1918.

22. Kries, Johannes von: Über Merkmale des Lebens. Freiburger Wissenschaftliche Gesellschaft. Heft 6. Freiburg 1919a.

23. Kries, Johannes von: Über Wahrscheinlichkeitsrechnung und ihre Anwendung in der Physik. In: Naturwiss. 7 (1919b), 2-7, 17-23.

24. Kries, Johannes von: Goethe als Naturforscher. Vortrag gehalten bei der Tagung der Goethe-Gesellschaft zu Weimar am 28. September 1919. In: Jahrb. d. Goethe-Ges. 7 (1920 a) 1-44.

25. Kries, Johannes von: Über die zwingende und eindeutige Bestimmtheit des physikalischen Weltbildes. In: Naturwiss. 8 (1920 b) 237-247.

26. Kries, Johannes von: von Helmholtz als Physiolog. In: Naturwiss. 9 (1921) 673-693.

27. Kries, Johannes von: Allgemeine Sinnesphysiologie. Leipzig 1923 a.

28. Kries, Johannes von: Zum Gedächtnis Karl Ludwigs. In: Naturwiss. 11 (1923 b) 1-4.

29. Kries, Johannes von: Goethe als Psycholog. In: Philosophie und Geschichte 5 (1924a) $1-52$.

30. Kries, Johannes von: Immanuel Kant und seine Bedeutung für die Naturforschung der Gegenwart. Berlin 1924b.

31. Kries, Johannes von: Kants Lehre von Zeit und Raum in ihrer Beziehung zur modernen Physik. In: Naturwiss. 12 (1924 c) 318-331.

32. Kries, Johannes von: Über logische Grenz- und Ausnahmefälle. In: Logos 13 (1924d) $31-66$.

33. Kries, Johannes von: Johannes von Kries. Die Medizin der Gegenwart in Selbstdarstellungen. Leipzig 1925.

34. Kries, Johannes von: Wer ist musikalisch? Gedanken zur Psychologie der Tonkunst. Berlin 1926.

35. Oser, Bertil Michael: Leben und Werk des Physiologen Johannes von Kries. Sinnesphysiologie und Erkenntniskritik. Med. Diss., Freiburg 1983.

36. Riehl, A.: von Helmholtz als Erkenntnistheoretiker. In: Naturwiss. 9 (1921) 702-708.

37. Rothschuh, Karl Eduard: Physiologie. Der Wandel ihrer Konzepte, Probleme und Methoden vom 16. bis 19. Jahrhundert. Freiburg/München 1968.

38. Rothschuh, Karl Eduard: History of Physiology. Translated and edited, with a new English bibliography by Guenter B. Risse. Huntington, New York 1973.

39. Schnädelbach, Herbert: Philosophie in Deutschland 1831-1933. Frankfurt am Main 1983.

40. Weizsäcker, Viktor von: Der Gestaltkreis. Leipzig 1940. Neuausgabe: Frankfurt am Main 1973. 
41. Weizsäcker, Viktor von: Gesammelte Schriften. Hrsg. von Peter Achilles, Dieter Janz, Martin Schrenk, Carl Friedrich von Weizsäcker. Bd.1 und 6. Frankfurt am Main 1986.

42. Wilhelm Windelband: Lehrbuch der Geschichte der Philosophie. Mit einem Schlußkapitel: Die Philosophie im 20. Jahrhundert und einer Übersicht über den Stand der philosophiegeschichtlichen Forschung. Hrsg. von Heinz Heimsoeth. Tübingen $1980^{17}$.

\section{Summary}

In the present study the attempt will be made to represent and compare the epistemiological import and implications for the philosophy of science with respect to the positions, found in the writings of the perception physiologists v. Helmholtz, v. Kries and v. Weizsäcker. In the continuity of the relation: teacher/student, it will at the same time be documented that the dialogue between philosophy and medicine in the scientific era has been pursued with various intentions and degrees of intensity.

v. Helmholtz maintained that the newly acquired integrity of scientific method must be defended. The need to show its import for medical practice lead him to reflect the relation of knowledge and practise from the fundament of his empirical theory and to define it as a linear relation of application. His student, v. Kries, realised that a deterministic principle for knowledge and practise could not meet the requirement for the self-determination of Man, and could not find a way using Neo-Kantianism influenced by psycho-physical parallelism to avoid this contradiction. First v. Weizsäcker dealt effectively with this problem and attempted to substitute causal thinking in medicine with the concept of «Gestaltkreis» intending to change medical practise and in this way to re-introduce Man in his subjectivity back into medicine. Thus, in the argumentation of three generations it can be demonstrated that the question concerning the relation between causality and freedom is considered as the decisive theme for the philosophy of science in modern medicine.

Dr. med. Josef Neumann

Institut für Geschichte der Medizin

Stefan-Meier-Straße 26

D-78 Freiburg im Breisgau 